\title{
Improved Cerebral Oxygen Saturation and Blood Flow Pulsatility With Pulsatile Perfusion During Pediatric Cardiopulmonary Bypass
}

\author{
XIAOWEI W. SU, YULONG GUAN, MOLLIE BARNES, J. BRIAN CLARK, JOHN L. MYERS, AND AKIF ÜNDAR
}

\author{
Departments of Pediatrics [X.W.S., Y.G., M.B., J.B.C., J.L.M., A.Ü.], Surgery [J.B.C., J.L.M., A.Ü.], and Bioengineering [A.Ü.], Penn \\ State Hershey College of Medicine, Penn State Hershey Children's Hospital, Hershey, Pennsylvania 17033
}

\begin{abstract}
Brain monitoring techniques near-infrared spectroscopy (NIRS) and transcranial Doppler (TCD) ultrasound were used in pediatric patients undergoing cardiopulmonary bypass for congenital heart defect (CHD) repair to analyze the effect of pulsatile or nonpulsatile flow on brain protection. Regional cerebral oxygen saturation $\left(\mathrm{rSO}_{2}\right)$ and cerebrovascular pulsatility index (PI) were measured by NIRS and TCD, respectively, in 111 pediatric patients undergoing bypass for CHD repair randomized to pulsatile $(n=77)$ or nonpulsatile $(n=34)$ perfusion. No significant differences in demographic and intraoperative data, including surgical risk stratification, existed between groups. Patients undergoing pulsatile perfusion had numerically lower decreases in $\mathrm{rSO}_{2}$ from baseline for all time points analyzed compared with the nonpulsatile group, with significant $\sim 12 \%$ lower decreases at 40 and 60 min after crossclamp. Patients undergoing pulsatile perfusion had numerically lower decreases in PI from baseline for the majority of time points compared with the nonpulsatile group, with significant $\sim 30 \%$ lower decreases between 5 and 40 min after crossclamp. Pulsatile flow has advantages over nonpulsatile flow as measured by NIRS and TCD, especially at advanced time points, which may improve postoperative neurodevelopmental outcomes. (Pediatr Res 70: 181-185, 2011)
\end{abstract}

A dvances in intraoperative surgical techniques and postoperative intensive care have substantively improved patient outcomes and decreased mortality rates in pediatric cardiac surgery for the repair of congenital heart defects (CHDs). With in-hospital mortality reported to be below $3 \%$ for categorized operations in a recent study despite an increase in case complexity (1), there is a growing need to address the paradoxical increased incidence of adverse neurological outcomes in the survivors of surgery. Cardiopulmonary bypass (CPB) presents unique challenges in this regard, with the extreme conditions encountered during extracorporeal support coupled with requisite surgical techniques that place severe stress on the patient acting in tandem to cause insult and injury to the brain. With increased incidence of postoperative neurologic complications reported in pediatric patients undergoing $\mathrm{CPB}$, including neuromotor disabilities, learning disabilities, and behavioral abnormalities $(2,3)$, there is increasing relevance for intra- and early postoperative brain monitoring

Received December 1, 2010; accepted February 26, 2011.

Correspondence: Akif Ündar, Ph.D., Department of Pediatrics, Surgery, and Bioengineering, Penn State Hershey College of Medicine, Department of Pediatrics-H085, 500 University Drive, P.O. Box 850, Hershey, PA 17033-0850; e-mail: aundar@psu.edu Supported by Children's Miracle Network [A.Ü.]. techniques, which may predict neurologic outcomes and provide medical professionals actionable information to aid in the minimization of adverse sequelae (4).

The debate between pulsatile and nonpulsatile perfusion modes further complicates considerations of best practices during pediatric $\mathrm{CPB}$ with the aim of minimizing neurological morbidity (5). Although current findings suggest that pulsatile flow imparts distinct advantages including increased hemodynamic energy leading to better blood flow to major organs such as brain, movement of lymph that prevents edema and sludging in microcapillaries, maintained microcirculatory flow, improved multiorgan function, and improved organ recovery $(6,7)$, there is little available evidence directly comparing pulsatile and nonpulsatile perfusion with regards to current brain monitoring techniques, CNS protection, and neurodevelopmental outcomes. In particular, comparisons of pediatric patients undergoing pulsatile and nonpulsatile perfusion using near-infrared spectroscopy (NIRS) to measure regional cerebral oxygen saturation $\left(\mathrm{rSO}_{2}\right)$ and transcranial Doppler (TCD) ultrasound to measure cerebrovascular pulsatility index (PI) are not available.

This study was conducted to address these limitations by analyzing NIRS and TCD measurements in pediatric patients receiving $\mathrm{CPB}$ for the repair of $\mathrm{CHD}$ randomized to pulsatile or nonpulsatile flow. Use of NIRS is supported by both animal and clinical data, as studies in a piglet model of CPB with deep hypothermic circulatory arrest (DHCA) showed the nadir of $\mathrm{rSO}_{2}$ occurred sooner at higher temperatures, lower hematocrits, and with $\alpha$-stat blood gas management, with time spent at nadir of $\mathrm{rSO}_{2}$ correlated with neurological outcome both behaviorally and histologically $(8,9)$; and in humans, lower intraoperative $\mathrm{rSO}_{2}$ levels during the myocardial ischemic period of CPB is associated with postoperative brain injury (10). TCD has been used extensively in pediatric cardiac surgery research to examine cerebrovascular hemodynamics under a variety of hypothermic and flow conditions. Alterations in cerebrovascular hemodynamics correlating with abnormal EEGs have been observed in pediatric patients

Abbreviations: CPB, cardiopulmonary bypass; CHD, congenital heart defect; MAP, mean arterial pressure; NIRS, near-infrared spectroscopy; PI, pulsatility index; $\mathbf{r S O}_{2}$, regional cerebral oxygen saturation; TCD, transcranial Doppler; XC, crossclamp 
undergoing profound hypothermia despite adequate perfusion using the technique (11), which has also proved useful in measuring levels of detectable blood flow during low-flow CPB (12) and hyperperfusion during complex surgical procedures (13). By comparing NIRS and TCD measurements in addition to standard operative variables in pediatric patients randomized to pulsatile or nonpulsatile perfusion, the relative merits of pulsatile flow in relation to brain injury and protection during CPB may be analyzed.

\section{MATERIALS AND METHODS}

Patients and study design. With Penn State IRB approval and parental informed consent, and under the guidance of a Data Safety Medical Board (DSMB) in addition to FDA clinicaltrials.com database registration, 238 patients were enrolled between March 2007 and February 2010 in a randomized trial comparing intra- and early postoperative physiologic measures as monitored by NIRS and TCD after receiving pulsatile or nonpulsatile perfusion during hypothermic CPB for surgical repair of CHDs. Eligibility criteria for subsequent statistical analysis included reparative surgery at $10 \mathrm{~d}$ to $5 \mathrm{y}$ $(1825 \mathrm{~d})$ of age and body weights between 2 and $17 \mathrm{~kg}$, yielding 145 subjects. Patients were excluded from subsequent statistical analysis for the following reasons: lack of complete NIRS data at the longest time points [40 and $60 \mathrm{~min}$ after crossclamp (XC)] when greatest changes were observed; lack of TCD data at the longest time points (40 and $60 \mathrm{~min}$ after XC); and lack of complete mean arterial pressure (MAP) data for all time points analyzed, leading to a final sample size of 111 patients of which 77 received pulsatile and 34 received nonpulsatile perfusion during $\mathrm{CPB}$. Cerebral oxygen saturation and cerebrovascular blood flow pulsatility monitoring was an integral part of the study design, with all patients analyzed receiving cerebral oxygen saturation and cerebrovascular blood flow pulsatility monitoring. In addition, MAPs were monitored throughout the surgical procedure to gauge general circulatory response.

Anesthesia and perfusion methods. Isoflurane and fentanyl facilitated with pancuronium was used to maintain anesthesia, and a median sternotomy was used for all operations. The CPB circuit used a Jostra HL-20 heart-lung machine (Jostra USA, Austin, TX) capable of pulsatile and nonpulsatile perfusion, a Stockert heart-cooler system (Sorin Group USA, Arvada, CO), Capiox hollow fiber membrane oxygenators, and a Capiox pediatric $32 \mu \mathrm{m}$ arterial filter (Terumo Cardiovascular Systems, Ann Arbor, MI). All circuit components are FDA approved and were selected based on previous studies demonstrating high-quality pulsatile perfusion (14). Circuit priming solution comprising lactated Ringer's, albumin and blood was provided. Target hematocrit during bypass was $25-27 \%$. Every patient received modified ultrafiltration. Pulsatile flow settings were maintained at a pump rate of 100 beats per minute, $10 \%$ of the base flow, $20 \%$ of the pump head start point, and $80 \%$ of the pump head stop point. These parameters were selected based on our pilot experiments for producing adequate pulsatility quality (14). No patient included in the study underwent DHCA.

Monitoring and data acquisition. $\mathrm{rSO}_{2}$ was measured using the INVOS 5100B (Somanetics, Troy, MI) NIRS monitor, which is FDA approved for pediatric use. The INVOS 5100B uses optimized algorithms that account for the thinner skull and extracranial tissues of infants and children and uses two near-infrared wavelengths (730 and $805 \mathrm{~nm}$ ) to measure the ratio of oxyhemoglobin to deoxyhemoglobin, which is expressed on a percent scale. After induction of anesthesia, Pediatric SomaSensors (Somanetics) were placed on the right and left forehead below the hairline according to the manufacturer's guidelines. A single sensor was used when space limitations precluded monitoring both hemispheres. Reported cerebral oxygen saturation values were the average of respective sensor readings when dual sensors were used or single sensor readings when a single sensor was used. After an initial accommodation period, NIRS data were collected at time intervals of $5 \mathrm{~s}$.

Cerebrovascular PI was measured using TCD ultrasound (Pioneer TC8080; Nicolet Biomedical Inc., Madison, WI). Sensors were placed on the right temporal window slightly anterior to the external auditory meatus and above the zygomatic arch. A 2-MHz pulsed-wave transducer was used to generate simultaneous $\mathrm{M}$-mode and spectrogram readings. Transtemporal insonation of the right middle cerebral artery (MCA) was conducted with insonation depth for spectrogram recordings between 25 and $50 \mathrm{~mm}$. Intraoperative NIRS and TCD data were recorded at the following time points: baseline preincision, on bypass before $\mathrm{XC}, 5 \mathrm{~min}$ after $\mathrm{XC}, 20 \mathrm{~min}$ after $\mathrm{XC}$, $40 \mathrm{~min}$ after XC, and $60 \mathrm{~min}$ after XC.

Statistical analysis. For this study, a sample size of 111 patients provided $80 \%$ power to detect a standardized effect size (i.e. the difference in means divided by a common SD) of 0.54 standard deviations using a two-sided test having a significance level of 0.05 , and $90 \%$ power to detect a standardized effect size of 0.62 standard deviations. Two-sample $t$ test was used to assess differences between pulsatile and nonpulsatile subjects for continuous variables; Mann Whitney $U$ test was used to assess differences in hospital course data because of skewness; Fisher's exact test was used to assess differences in categorical data. All hypothesis tests were two-sided, and all analysis was performed using StatView 5 for Windows (SAS Institute, Inc., Cary, NC), with significance set at $p<0.05$. All data are presented as mean \pm SEM unless otherwise noted.

\section{RESULTS}

All patients survived to discharge, and none developed clinical seizures, stroke, or choreoathetosis. Demographic and operative characteristics according to randomized perfusion mode are shown in Table 1. There were no significant differences in age, weight, or gender between groups. Risk categories of Jenkins et al. (15) were not different between groups. Intraoperative characteristics including total CPB (pump)

Table 1. Preoperative and operative characteristics

\begin{tabular}{|c|c|c|c|}
\hline Variable & Pulsatile & Nonpulsatile & $p$ \\
\hline \multicolumn{4}{|l|}{ Patient characteristics } \\
\hline Age (d), mean (range) & $351(13-1810)$ & $427(12-1798)$ & 0.125 \\
\hline Weight $(\mathrm{kg})$, mean (range) & $7.1(2-16.9)$ & $7.8(2-15.4)$ & 0.297 \\
\hline Gender (\% male) & 57.1 & 44.1 & 0.222 \\
\hline \multicolumn{4}{|c|}{ Surgical and intraoperative characteristics } \\
\hline Jenkins risk category, mean & $2.3 \pm 0.1$ & $2.3 \pm 0.1$ & 0.969 \\
\hline Total CPB time (min) & $113 \pm 4$ & $116 \pm 8$ & 0.662 \\
\hline $\mathrm{XC}$ time $(\min )$ & $81 \pm 2$ & $74 \pm 6$ & 0.187 \\
\hline Nadir temperature $\left({ }^{\circ} \mathrm{C}\right)$ & $30.6 \pm 0.3$ & $31.0 \pm 0.5$ & 0.463 \\
\hline Mean flow rate $(\mathrm{mL} / \mathrm{min})$ & $920 \pm 41$ & $977 \pm 62$ & 0.449 \\
\hline \multicolumn{4}{|c|}{ MAP_-change from baseline (numerical values, $\mathrm{mm} \mathrm{Hg}$ ) } \\
\hline Baseline & $60.8 \pm 1.7$ & $63.7 \pm 2.2$ & 0.315 \\
\hline Before XC & $-15.6 \pm 2.5$ & $-21.3 \pm 3.1$ & 0.182 \\
\hline 5 min after $\mathrm{XC}$ & $-23.8 \pm 2.0$ & $-24.5 \pm 2.6$ & 0.846 \\
\hline 20 min after $\mathrm{XC}$ & $-20.6 \pm 1.9$ & $-22.4 \pm 2.1$ & 0.585 \\
\hline 40 min after $\mathrm{XC}$ & $-18.5 \pm 1.9$ & $-21.3 \pm 2.3$ & 0.386 \\
\hline 60 min after $\mathrm{XC}$ & $-17.0 \pm 2.1$ & $-19.9 \pm 2.9$ & 0.431 \\
\hline
\end{tabular}

Risk category of Jenkins et al. (15). 
time, XC time, nadir temperature, and average flow rates were not different between groups. The pulsatile group experienced numerically lower decreases in MAP from baseline at all time points compared with the nonpulsatile group, although results were no statistically significant. Group sizes were $<77$ and 34 patients for pulsatile and nonpulsatile groups, respectively, in select measures owing to lack of data for respective patients. Complete data for all patients were available for the majority of measures for combined cerebral oxygen saturation and cerebrovascular pulsatility, and all measures for MAP.

Pulsatile versus nonpulsatile waveforms. Representative blood flow waveforms from pulsatile and nonpulsatile groups are presented in Figure 1. TCD was used to record cerebral blood flow waveforms generated by pulsatile and nonpulsatile flow settings, which were analyzed computationally to yield Gosling's PI values for subsequent statistical analysis. Previous research has shown the challenges of generating pulsatile flow with small diameter aortic cannulae (16); PI data presented below enables quantitative assessment of the quality of pulsatile perfusion attained.

Changes in cerebral oxygen saturation. The group receiving pulsatile $\mathrm{CPB}$ perfusion experienced numerically lower decreases in $\mathrm{rSO}_{2}$ levels from baseline at all time points
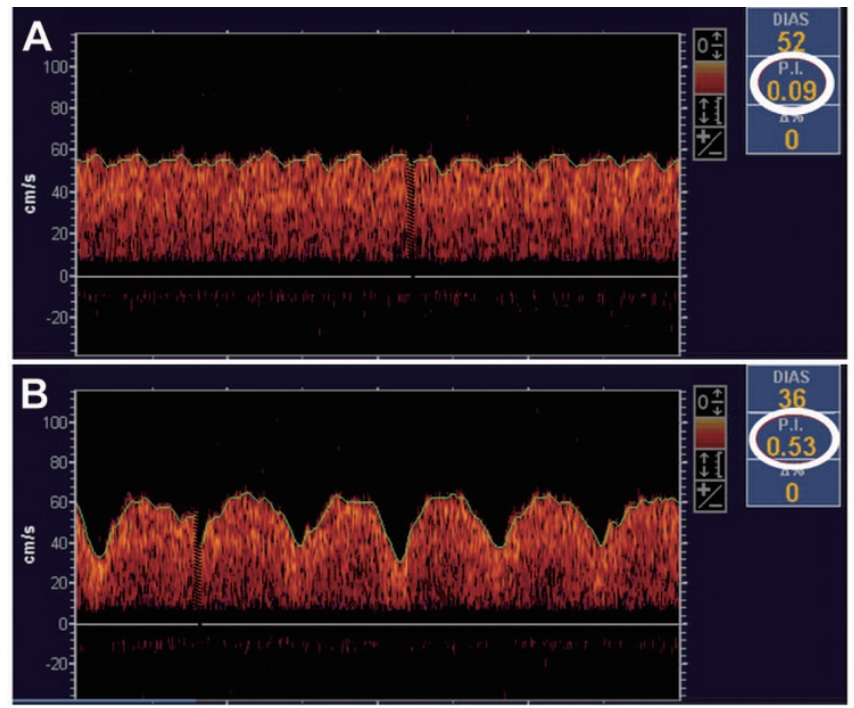

Figure 1. Representative blood flow waveforms obtained using TCD from nonpulsatile $(A)$ and pulsatile $(B)$ groups. PI readings are circled in white. analyzed compared with the nonpulsatile group as measured by both absolute and percent change (Figs. $2 A$ and $B$, respectively). At the longest time points sampled, 40 and $60 \mathrm{~min}$ after XC, the pulsatile group experienced significantly lower decreases in $\mathrm{rSO}_{2}$ compared with the nonpulsatile group as measured by percent change $(p=0.026$ and $p=0.044$, respectively; Fig. $2 B$ and Table 2 ).

Changes in cerebrovascular PI. TCD data reported as Gosling's PI, which reflects cerebrovascular hemodynamics, is presented as change from baseline and is given in Figures $3 A$ and $B$ and Table 2. The pulsatile group experienced numerically lower decreases in PI from baseline at all time points except before XC compared with the nonpulsatile group as measured by both absolute and percent change (Figs. $3 A$ and $B$ ). The pulsatile group experienced a significantly lower absolute decrease in PI from baseline measured $20 \mathrm{~min}$ after XC ( $p=0.010$; Fig. 3A). The pulsatile group experienced significantly lower percentage decreases in PI from baseline between 5 and $40 \mathrm{~min}$ after $\mathrm{XC}$ compared with the nonpulsatile group $(p=0.016, p<0.001$ and $p<0.001$, respectively; Fig. $3 B$ ). The latter group experienced a $\sim 30 \%$ greater decrease in PI at these time points.

\section{DISCUSSION}

NIRS and TCD monitoring possess validity in assessing brain oxygen saturation and cerebrovascular blood flow pulsatility, respectively, and are relevant to the prediction and prevention of neurological morbidity subsequent to CPB. Studies have also shown that patients experiencing low $\mathrm{rSO}_{2}$ levels during pediatric CPB experience adverse acute neurological outcomes including prolonged coma, seizures, or hemiparesis $(10,17)$. TCD is commonly used to assess cerebral hemodynamics during CPB (18) and has been shown to be accurate and reliable in infants and young children (19). Alterations in cerebrovascular hemodynamics is reported in patients experiencing profound hypothermia (20), a condition known to cause adverse neurological outcomes (21). In addition, the relative homogeneity of the patient population in this study with regards to operative variables such as methods of anesthesia, extracorporeal circuit components, and pulsatile and nonpulsatile pump settings, respectively, further add to the reliability of results presented. Take together these factors argue for the soundness of current study methodology and validity of present results.
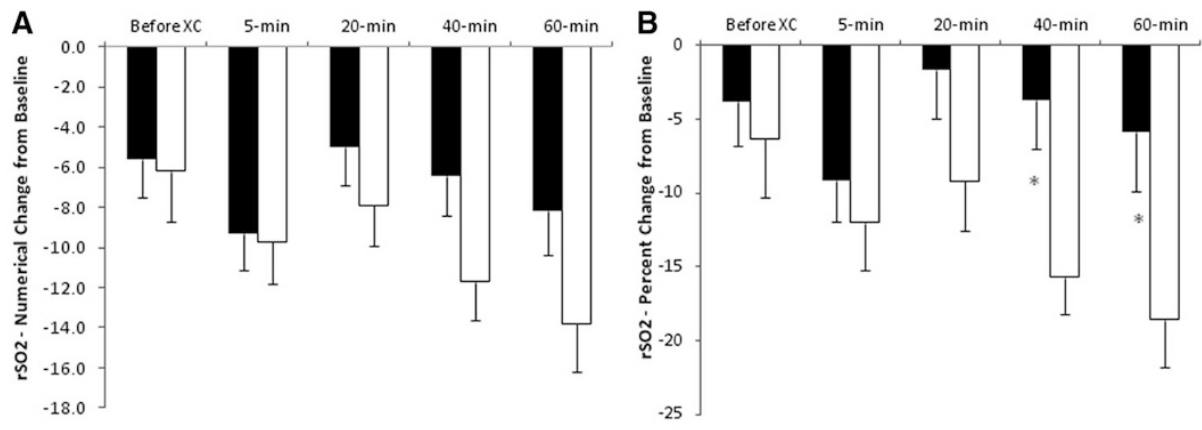

Figure 2. Regional cerebral oxygenation as measured by NIRS. Data are presented as numerical change from baseline $(A)$ and percent change from baseline $(B) . * p<$ 0.05 (ם: pulsatile flow; $\square$ : nonpulsatile flow). 
Table 2. Regional cerebral oxygen saturation and pulsatility index results

\begin{tabular}{|c|c|c|c|}
\hline Variable & Pulsatile & Nonpulsatile & $p$ \\
\hline \multicolumn{4}{|c|}{$\mathrm{rSO}_{2}$ - change from baseline (numerical values, $\mathrm{O}_{2}$ saturation \%) } \\
\hline Baseline & $66.2 \pm 2.0$ & $68.9 \pm 2.0$ & 0.409 \\
\hline Before XC & $-5.6 \pm 1.9$ & $-6.2 \pm 2.5$ & 0.829 \\
\hline 5 min after $\mathrm{XC}$ & $-9.3 \pm 1.8$ & $-9.7 \pm 2.1$ & 0.917 \\
\hline $20 \mathrm{~min}$ after $\mathrm{XC}$ & $-5.0 \pm 1.9$ & $-7.9 \pm 2.0$ & 0.358 \\
\hline 40 min after $\mathrm{XC}$ & $-6.4 \pm 2.0$ & $-11.7 \pm 1.9$ & 0.109 \\
\hline 60 min after $\mathrm{XC}$ & $-8.2 \pm 2.2$ & $-13.8 \pm 2.4$ & 0.122 \\
\hline \multicolumn{4}{|l|}{$\mathrm{rSO}_{2}$ - change from baseline $(\%)$} \\
\hline Before XC & $-3.8 \pm 3.0$ & $-6.3 \pm 4.0$ & 0.631 \\
\hline 5 min after $\mathrm{XC}$ & $-9.2 \pm 2.7$ & $-12.0 \pm 3.2$ & 0.553 \\
\hline 20 min after $\mathrm{XC}$ & $-1.7 \pm 3.2$ & $-9.2 \pm 3.3$ & 0.161 \\
\hline 40 min after $\mathrm{XC}$ & $-3.7 \pm 3.3$ & $-15.7 \pm 2.5$ & $0.026^{*}$ \\
\hline 60 min after $\mathrm{XC}$ & $-5.9 \pm 4.0$ & $-18.6 \pm 3.2$ & $0.044^{*}$ \\
\hline \multicolumn{4}{|c|}{ Pulsatility index — change from baseline (numerical values, PI) } \\
\hline Baseline & $1.46 \pm 0.06$ & $1.45 \pm 0.10$ & 0.917 \\
\hline Before XC & $-0.76 \pm 0.08$ & $-0.66 \pm 0.12$ & 0.500 \\
\hline 5 min after $\mathrm{XC}$ & $-0.56 \pm 0.10$ & $-0.84 \pm 0.13$ & 0.106 \\
\hline 20 min after $\mathrm{XC}$ & $-0.48 \pm 0.08$ & $-0.88 \pm 0.12$ & $0.010^{*}$ \\
\hline 40 min after $\mathrm{XC}$ & $-0.52 \pm 0.10$ & $-0.83 \pm 0.08$ & 0.056 \\
\hline 60 min after $\mathrm{XC}$ & $-0.54 \pm 0.10$ & $-0.63 \pm 0.12$ & 0.614 \\
\hline \multicolumn{4}{|c|}{ Pulsatility index-change from baseline $(\%)$} \\
\hline Before XC & $-47.7 \pm 4.3$ & $-37.6 \pm 10.6$ & 0.292 \\
\hline 5 min after $\mathrm{XC}$ & $-31.3 \pm 6.4$ & $-57.7 \pm 6.1$ & $0.016^{*}$ \\
\hline 20 min after $\mathrm{XC}$ & $-28.8 \pm 5.5$ & $-60.6 \pm 4.8$ & $0.001^{*}$ \\
\hline $40 \mathrm{~min}$ after $\mathrm{XC}$ & $-28.5 \pm 5.9$ & $-61.6 \pm 4.8$ & $0.001^{*}$ \\
\hline 60 min after $\mathrm{XC}$ & $-33.1 \pm 5.8$ & $-44.5 \pm 8.1$ & 0.262 \\
\hline
\end{tabular}

$* p<0.05$ vs. baseline.
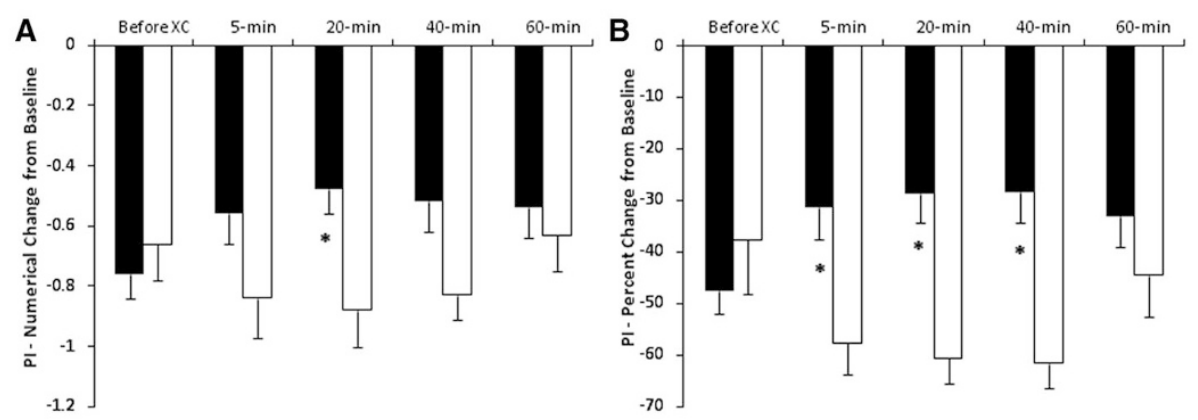

Figure 3. Gosling's PI as measured by TCD. Data are presented as numerical change from baseline $(A)$ and percent change from baseline $(B)$. ${ }^{*} p<0.05$ pulsatile flow; $\square$ : nonpulsatile flow).

This study extends previous work on the benefits of pulsatile versus nonpulsatile perfusion during pediatric CPB for the repair of CHD with two significant findings. First, findings demonstrate that pulsatile flow results in numerically lower decreases in cerebral oxygen saturation levels compared with nonpulsatile flow at all time points analyzed as measured by NIRS, with statistically significant increases occurring at the longest time points, 40 and $60 \mathrm{~min}$ after XC. Second, findings demonstrate that pulsatile flow results in numerically lower decreases in cerebrovascular PI as measured by TCD in the majority of time points measured, with significantly lower decreases at time points between 5 and 40 min after XC, inclusive. At these time points onward, pulsatile flow resulted in a $\sim 30 \%$ lower decrease in PI compared with nonpulsatile flow, which may promote improved cerebrovascular hemodynamics and recovery $(6,7)$.

The absence of significant differences between pulsatile and nonpulsatile groups at initial time points suggests that, at least with regards to brain injury, relatively milder operative stress occurs early into $\mathrm{CPB}$, masking differences between the respective perfusion modes. However, with increasing time spent under XC, significant differences in $\mathrm{rSO}_{2}$ and PI values emerge, with a consistent benefit of pulsatile flow seen in all measures analyzed at advanced time points. Studies have reported increasing rates of adverse acute and neurodevelopmental outcomes in patients experiencing longer times under hypothermic $\mathrm{CPB}$, which is exacerbated by worsening operative conditions posing greater challenges for surgical procedures in general at these time points $(22,23)$, and as such methods that may ameliorate adverse events during long operative protocols are of great relevance to brain protection. Results of this study provide further evidence of the benefits of pulsatile over nonpulsatile flow by demonstrating improved readings in brain monitoring modalities that represent novel measures in the context of perfusion mode comparisons.

Although present findings support the conclusion that pulsatile flow has distinct advantages over nonpulsatile flow in brain protection during $\mathrm{CPB}$, at least at advanced time points 
during long protocols, results are limited by certain shortcomings in experimental design and data collection. Space limitations on the forehead of certain patients during surgery precluded the use of dual NIRS sensors in these patients, leading to reliance on data from a single sensor. This study did not use newer monitoring modalities such as visible light spectroscopy (VLS) (24) or emboli detection and classification (EDAC) quantification (25), measures which may have enhanced results obtained. TCD data indicated that nonpulsatile rather than pulsatile perfusion resulted a lower decrease in $\mathrm{PI}$ at the before XC time point, which may possibly have been because of the variances in hemodynamic stability as surgical teams prepared for $\mathrm{XC}$ administration.

Percent changes were reported for $\mathrm{rSO}_{2}$ and $\mathrm{PI}$ results when cross comparisons were conducted because each patient had a different respective baseline value. In addition, to comparing one group with another, we were also able to compare each patient with his/her baseline values and determine whether there was a significant change within the same patient, within the same group, and between groups.

Follow-up studies to monitor neurological outcomes are currently ongoing in the patient population analyzed in this study. By correlating readings from the respective brain monitoring modalities recorded here with neurodevelopmental outcomes, including measures of cognitive and speech impairments, motor deficits, behavioral abnormalities, and educational achievement, and taking into consideration the contribution of pulsatile versus nonpulsatile flow to outcomes, it is hoped that the neurodevelopmental benefits of pulsatile perfusion techniques may be ascertained.

A growing body of research supports the conclusion that pulsatile perfusion has distinct advantages over nonpulsatile perfusion during pediatric CPB (4-7). This study adds to this literature by demonstrating a benefit of pulsatile flow to cerebral oxygen saturation and blood flow pulsatility during surgery for CHD, which may lead to decreased incidence of neurological sequelae and improved neurodevelopmental outcomes subsequent to $\mathrm{CPB}$.

\section{REFERENCES}

1. Welke KF, Shen I, Ungerleider RM 2006 Current assessment of mortality rates in congenital cardiac surgery. Ann Thorac Surg 82:164-170; discussion 170-171

2. Gessler P, Schmitt B, Pretre R, Latal B 2009 Inflammatory response and neurodevelopmental outcome after open-heart surgery in children. Pediatr Cardiol 30:301305

3. Wernovsky G, Shillingford AJ, Gaynor JW 2005 Central nervous system outcomes in children with complex congenital heart disease. Curr Opin Cardiol 20:94-99

4. Su XW, Undar A 2010 Brain protection during pediatric cardiopulmonary bypass. Artif Organs 34:E91-E102
5. Ündar A 2005 Pulsatile versus nonpulsatile cardiopulmonary bypass procedures in neonates and infants: from bench to clinical practice. ASAIO J 51:vi-X

6. Akçevin A, Alkan-Bozkaya T, Qiu F, Ündar A 2010 Evaluation of perfusion modes on vital organ recovery and thyroid hormone homeostasis in pediatric patients undergoing cardiopulmonary bypass. Artif Organs 34:879-884

7. Haines N, Wang S, Ündar A, Alkan T, Akcevin A 2009 Clinical outcomes of pulsatile and non-pulsatile mode of perfusion. J Extra Corpor Technol 41:P26-P29

8. Sakamoto T, Zurakowski D, Duebener LF, Hatsuoka S, Lidov HG, Holmes GL, Stock UA, Laussen PC, Jonas RA 2002 Combination of alpha-stat strategy and hemodilution exacerbates neurologic injury in a survival piglet model with deep hypothermic circulatory arrest. Ann Thorac Surg 73:180-189; discussion 189-190

9. Sakamoto T, Hatsuoka S, Stock UA, Duebener LF, Lidov HG, Holmes GL, Sperling JS, Munakata M, Laussen PC, Jonas RA 2001 Prediction of safe duration of hypothermic circulatory arrest by near-infrared spectroscopy. J Thorac Cardiovasc Surg 122:339-350

10. McQuillen PS, Barkovich AJ, Hamrick SE, Perez M, Ward P, Glidden DV, Azakie A, Karl T, Miller SP 2007 Temporal and anatomic risk profile of brain injury with neonatal repair of congenital heart defects. Stroke 38:736-741

11. O’Hare B, Bissonnette B, Bohn D, Cox P, Williams W 1995 Persistent low cerebral blood flow velocity following profound hypothermic circulatory arrest in infants Can J Anaesth 42:964-971

12. Zimmerman AA, Burrows FA, Jonas RA, Hickey PR 1997 The limits of detectable cerebral perfusion by transcranial Doppler sonography in neonates undergoing deep hypothermic low-flow cardiopulmonary bypass. J Thorac Cardiovasc Surg 114:594600

13. Andropoulos DB, Stayer SA, McKenzie ED, Fraser CD Jr 2003 Novel cerebral physiologic monitoring to guide low-flow cerebral perfusion during neonatal aortic arch reconstruction. J Thorac Cardiovasc Surg 125:491-499

14. Wang S, Haines N, Ündar A 2009 Quantification of pressure-flow waveforms and selection of components for the pulsatile extracorporeal circuit. J Extra Corpor Technol 41:P20-P25

15. Jenkins KJ, Gauvreau K, Newburger JW, Spray TL, Moller JH, Iezzoni LI 2002 Consensus-based method for risk adjustment for surgery for congenital heart disease. J Thorac Cardiovasc Surg 123:110-118

16. Rider AR, Ji B, Kunselman AR, Weiss WJ, Myers JL, Ündar A 2008 A performance evaluation of eight geometrically different $10 \mathrm{Fr}$ pediatric arterial cannulae under pulsatile and nonpulsatile perfusion conditions in an infant cardiopulmonary bypass model. ASAIO J 54:306-315

17. Austin EH III, Edmonds HL Jr, Auden SM, Seremet V, Niznik G, Sehic A, Sowell MK, Cheppo CD, Corlett KM 1997 Benefit of neurophysiologic monitoring for pediatric cardiac surgery. J Thorac Cardiovasc Surg 114:707-715, 717; discussion 715-716

18. Polito A, Ricci Z, Di Chiara L, Giorni C, Iacoella C, Sanders SP, Picardo S 2006 Cerebral blood flow during cardiopulmonary bypass in pediatric cardiac surgery: the role of transcranial Doppler-a systematic review of the literature. Cardiovasc Ultrasound 4:47

19. Rogerson A, Guan Y, Kimatian SJ, Kunselman A, Clark JB, Myers JL, Ündar A 2010 Transcranial Doppler ultrasonography: a reliable method of monitoring pulsatile flow during cardiopulmonary bypass in infants and young children. J Thorac Cardiovasc Surg 139:e80-e82

20. Jonassen AE, Quaegebeur JM, Young WL 1995 Cerebral blood flow velocity in pediatric patients is reduced after cardiopulmonary bypass with profound hypothermia. J Thorac Cardiovasc Surg 110:934-943

21. Williams GD, Ramamoorthy C 2007 Brain monitoring and protection during pediatric cardiac surgery. Semin Cardiothorac Vasc Anesth 11:23-33

22. Wypij D, Newburger JW, Rappaport LA, duPlessis AJ, Jonas RA, Wernovsky G, Lin M, Bellinger DC 2003 The effect of duration of deep hypothermic circulatory arrest in infant heart surgery on late neurodevelopment: the Boston Circulatory Arrest Trial. J Thorac Cardiovasc Surg 126:1397-1403

23. Hövels-Gürich HH, Konrad K, Skorzenski D, Nacken C, Minkenberg R, Messmer BJ, Seghaye MC 2006 Long-term neurodevelopmental outcome and exercise capacity after corrective surgery for tetralogy of Fallot or ventricular septal defect in infancy. Ann Thorac Surg 81:958-966

24. Benaron DA, Parachikov IH, Friedland S, Soetikno R, Brock-Utne J, van der Starre PJ, Nezhat C, Terris MK, Maxim PG, Carson JJ, Razavi MK, Gladstone HB, Fincher EF, Hsu CP, Clark FL, Cheong WF, Duckworth JL, Stevenson DK 2004 Continuous, noninvasive, and localized microvascular tissue oximetry using visible light spectroscopy. Anesthesiology 100:1469-1475

25. Wang S, Woitas K, Clark JB, Myers JL, Ündar A 2009 Clinical real-time monitoring of gaseous microemboli in pediatric cardiopulmonary bypass. Artif Organs 33:1026-1030 